\title{
Welche Methode liefert zuverlässige Werte?
}

Fragestellung: Kann ein auf fetales Hämoglobin (HbF) adjustierter $\mathrm{HbA}_{1 \mathrm{c}}$ im Vergleich zum glykierten Albumin oder dem normalen $\mathrm{HbA}_{1 \mathrm{c}}$ ein guter Indikator für den Glukosestoffwechsel von Säuglingen und Neugeborenen sein?

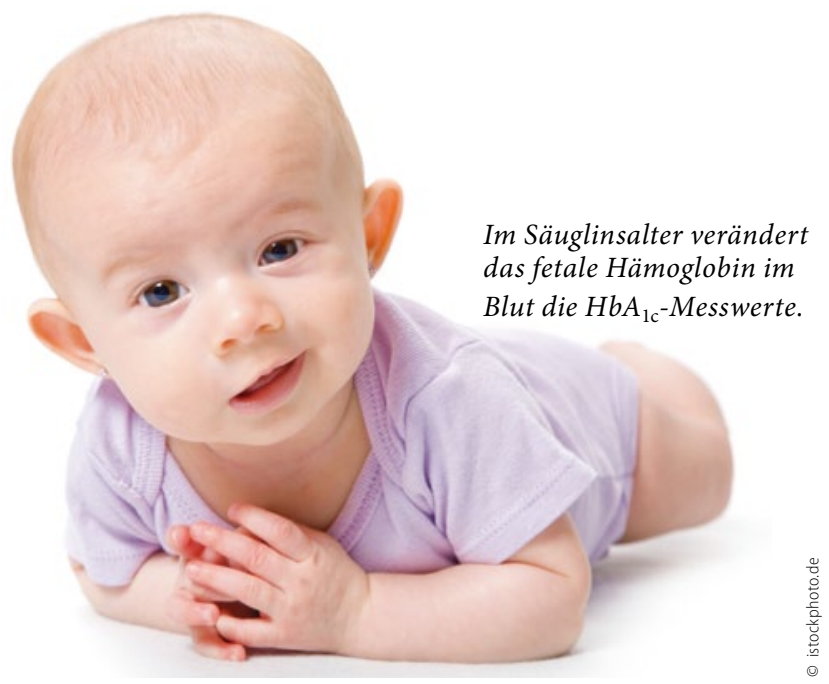

Hintergrund: Die Glykierung von Eiweißen ist bei Diabetiker höher als bei Gesunden. Das wird genutzt, um die Diagnose Diabetes stellen und die Therapie überwachen zu können. Typische Parameter sind der $\mathrm{HbA}_{1 c}$, Fructosamin oder glykiertes Albumin (GA). Für $\mathrm{HbA}_{1 \mathrm{c}}$-Messungen sind Veränderungen der Zusammensetzung des Blutes bezügliche der Hämoglobinvarianten störend. Innerhalb der ersten Lebensmonate wird die Produktion von hauptsächlich fetalem $(\mathrm{HbF})$ auf adultes Hämoglobin (HbA) umgestellt. Sind die HbF-Spiegel hoch und die HbA-Spiegel niedrig, wird ein falsch niedriger $\mathrm{HbA}_{1 \mathrm{c}}$ gemessen. Daher scheint insbesondere im Neugeborenen- und frühen Säuglingsalter die Bestimmung des $\mathrm{HbA}_{\mathrm{cc}}$ zur Stoffwechselkontrolle bei neonatalem Diabetes wenig sinnvoll.
Patienten und Methodik: Blutproben von 26 gesunden Neugeborenen oder Säuglingen (4-234 Tage alt) wurden bei einem Neugeborenenscreening oder einem elektiven Eingriff entnommen. Plasmaglukose (präprandial, nicht immer nüchtern) und der $\mathrm{HbA}_{1 \mathrm{c}}$-Wert (mithilfe der Hochdruckflüssigchromatografie, HPLC) wurden bestimmt. Zudem erfasste man mithilfe einer immunologischen Methode auch das glykierte Hämoglobin. Um den Einfluss des $\mathrm{HbF}$ herauszurechnen, wurde der $\mathrm{HbA}_{\mathrm{lc}}$ daraufhin adjustiert $\left(\mathrm{HbA}_{1 \mathrm{c}} /\right.$ Gesamthämoglobin-fetales Hämoglobin). Zusätzlich bestimmte man das GA.

Ergebnisse: $\mathrm{HbF}$ war bis zum 8. Lebensmonat (LM) nachweisbar, bis zum 6. LM in $\mathrm{HbA}_{1 \mathrm{c}}$ sicher beeinflussender Höhe (15\%). $\mathrm{HbA}_{1 \mathrm{c}}$-Werte ohne Korrektur waren signifikant niedriger als die für den HbF-Anteil korrigierten Werte (immunologisch: 2,7 $\pm 1,3 \%$, HPLC: $2,7 \pm 1,3 \%$; Adjustiert mit HPLC: $4,7 \pm 0,6 \%$, adjustiert mit immunologischer Methode: 4,5 $\pm 0,5 \%$ ). Verwendet man die übliche nicht korrigierte Methode, liegen die $\mathrm{HbA}_{1 c^{-}}$ Werte bei Säuglingen im Alter unter sechs Monaten signifikant niedriger als der Normbereich im Alter von 8-12 Monaten. Die GA-Spiegel korrelierten sehr gut mit der Plasmaglukose. Der adjustierte $\mathrm{HbA}_{1 \mathrm{c}}$ (egal welche Methode) korrelierte aber nur gering mit der Plasmaglukose. Es zeigte sich eine gute Korrelation von GA mit den korrigierten, adjustierten $\mathrm{HbA}_{1 \mathrm{c}}$-Werten, die mit der immunologischen Methode ermittelt wurden, wohingegen die adjustierten HPLC-Messwerte nicht korrelierten.

Schlussfolgerung: Die immunologische $\mathrm{HbA}_{1 \mathrm{c}}$-Messung spiegelt im Säuglingsalter recht zuverlässig den Glukosestoffwechsel wider, wenn der $\mathrm{HbA}_{1 \mathrm{c}}$ für $\mathrm{HbF}$ korrigiert wird; die HPLC-Methode, auch wenn adjustiert, ist eher unzuverlässig.
Suzuki S, Koga M, Niizeki N et al. Evaluation of glycated hemoglobin and fetal hemoglobinadjusted $\mathrm{HbA1c}$ measurements in infants. Pediatric Diabetes 2013;14:267-72.

\section{- Kommentar von PD Dr. med. Thomas Kapellen}

\section{Hämoglobinmauser macht die Sache knifflig}

Das GA wurde in dieser Studie zum Vergleich herangezogen, da hier Normwerte auch für Säuglinge vorliegen. Es wird nicht von der "Hämoglobinmauser" des Säuglings beeinflusst und korreliert sehr gut mit Plasmaglukoseprofilen. Der Schwachpunkt der Studie ist, dass nur ein Plasmaglukosewerte präprandial, aber nicht sicher immer nüchtern am Morgen bestimmt wurde. Besser für die Aussagekraft wäre ein Profil oder wären zumindest mehrere Werte gewesen. Dennoch zeigt sich - wie erwartet - die Schwäche der $\mathrm{HbA}_{1 c}$-Messung in Abhängigkeit vom $\mathrm{HbA}$-Gehalt des Blutes, der mindestens bis zum 6 . Lebensmonat relevant ist. Am besten mit dem GA korrelierten adjustierte $\mathrm{HbA}_{1 \mathrm{c}}$-Werte mittels immunologischer Bestim- mung. Solche adjustierten $\mathrm{HbA}_{1 \mathrm{c}}$-Werte sind schwierig in die klinische Routine einzubinden, da man immer das $\mathrm{HbF}$ bestimmen muss. Auch HPLC-Methoden, die HbF eliminieren, sind bislang nicht adäquat dahingehend überprüft worden, ob die Messwerte im Säuglingsalter zuverlässig sind. Die Autoren schlussfolgern aber für mich nicht ganz verständlich daraus nicht, dass alternativ eine $\mathrm{Hb}$-unabhängige Methode, wie Fructosamin oder GA, zu favorisieren ist. Bleibt nur der Weg über eine Adjustierung oder mehrfache Glukosemessungen oder gar eine kontinuierliche Glukosemessung bei Säuglingen, die jünger als sechs Monate sind.

PD Dr. med. Thomas M. Kapellen, Leipzig 